\title{
Circuit
}

Musiques contemporaines

\section{Interprète et créateur}

Entretien avec Benjamin Carat

Performer/Composer

\section{Interview with Benjamin Carat}

\section{Réjean Beaucage}

Volume 15, numéro 1, 2004

Interpréter la musique (d')aujourd'hui

URI : https://id.erudit.org/iderudit/902342ar

DOI : https://doi.org/10.7202/902342ar

Aller au sommaire du numéro

Éditeur(s)

Les Presses de l'Université de Montréal

ISSN

1183-1693 (imprimé)

1488-9692 (numérique)

Découvrir la revue

Citer ce document

Beaucage, R. (2004). Interprète et créateur : entretien avec Benjamin Carat. Circuit, 15(1), 65-72. https://doi.org/10.7202/902342ar
Résumé de l'article

Le violoncelliste français Benjamin Carat privilégie l'interprétation d'oeuvres nouvelles issues d'une étroite collaboration avec les compositeurs durant le processus de création. S'employant à développer un nouveau répertoire pour son instrument, il concentre son travail sur la musique mixte et les nouvelles techniques de jeu. Parcours biographique et réflexion sur le métier d'interprète. d'utilisation que vous pouvez consulter en ligne.

https://apropos.erudit.org/fr/usagers/politique-dutilisation/ 


\title{
Interprète et créateur
}

\author{
Entretien avec Benjamin Carat
}

Réjean Beaucage

LE VIOLONCELliste FRANÇAIS Benjamin Carat s'emploie depuis quelques années à développer un nouveau répertoire pour son instrument en concentrant son travail sur la musique mixte et les nouvelles techniques de jeu. Dans l'entretien qui suit, j'explore avec lui le parcours biographique qui l'a amené à faire ce choix et nous discutons des rapports entre interprète et compositeur ou entre interprétation et création.

BENJAMIn CARAT : J'ai commencé très jeune à jouer du violoncelle, on ne peut donc pas dire que ce soit un choix personnel. Ma mère est pianiste et mon père, sans être musicien (bien qu'il chante dans des chorales amateurs), est mélomane. Tous les deux aimaient bien l'instrument et connaissaient un professeur, vers lequel ils m'ont dirigé à l'âge de cinq ans. Alors évidemment, à ce moment-là, on joue sur un seizième, puis un huitième, un quart, un demi, et finalement un violoncelle «entier ». Mon père m'a acheté un violoncelle entier lorsque j'ai eu 14 ans et c'est à ce moment-là que j'ai rencontré, à l'occasion de l'Académie d'été, d'autres violoncellistes que mon professeur, le seul que je connaissais, qui était très bien, mais tout de même, il était le seul. J'ai ainsi rencontré le violoncelliste principal de l'Orchestre de l'Opéra Bastille qui m'a fait entendre un tout autre violoncelle que celui que je connaissais, extrêmement virtuose; et ces cours m'ont suffisamment impressionné pour que je 
décide de m'investir complètement dans la pratique instrumentale. Comme j'avais commencé assez tôt les études, j'avais pratiquement déjà terminé le Conservatoire à cet âge-là, je pouvais songer aux conservatoires supérieurs et choisir l'option professionnelle.

Après le Conservatoire d'Annecy, je suis allé à Lyon où je suis entré à 18 ans au Conservatoire National Supérieur de Musique (CNSM). J'en suis sorti quatre ans plus tard et j'ai fait la rencontre du compositeur Robert Pascal, qui a eu une influence considérable sur mon parcours parce qu'il donnait des cours d'analyse d'œuvres du $\mathrm{XX}^{\mathrm{e}}$ siècle que nous devions ensuite interpréter en concert. Ça a été extraordinaire, il m'a fait découvrir ce répertoire. Et puis c'était une personnalité intéressante parce qu'il est aussi agrégé de mathématiques et qu'il animait au Conservatoire une classe d'informatique musicale destinée aux instrumentistes; j'étais le seul à m'y être inscrit! Alors il s'est bien occupé de moi et, en conséquence, je me suis rapproché de l'informatique. À mon concours de sortie, il y avait un programme établi à présenter qui comprenait suite de Bach, sonate de Beethoven et concerto de Schumann ainsi qu'une œuvre au choix. Ça m'a permis de faire une petite révolution au Conservatoire en présentant Chant d'Aubes, de Robert Pascal, une œuvre pour violoncelle avec dispositif électronique fonctionnant en temps réel. Ça a été un peu compliqué pour la mise en œuvre parce que c'était plus difficile qu'aujourd'hui, technologiquement (en 1997!). J'ai interprété cette œuvre au moins une cinquantaine de fois jusqu'à maintenant.

Le fait d'avoir rencontré Robert Pascal m'a aussi rapproché du GRAME (Centre National de Création Musicale situé à Lyon), qui m'a offert à plusieurs occasions de jouer en concert. Par exemple, le premier concert que j'ai fait avec le GRAME était la création française d'une pièce de Jonathan Harvey, Louanges, pour chœur et violoncelle solo; petit à petit, ma spécificité d'interprète de musique mixte s'est développée au GRAME. En sortant du CNSM, j’ai fait un an à l'IRCAM pour ma première année d'études doctorales (DEA) et j'ai rédigé un mémoire dont le titre était Le nouveau rapport interprète/instrument dans les œuvres pour violoncelle nécessitant un dispositifélectronique.

R. B. : Si l'on pense à la pièce de Robert Pascal, où l'interprète interagit avec un dispositif assez important, peut-on s'attendre à entendre la même pièce chaque fois?

B. C. : Non, c'est une pièce totalement interactive et je l'aime beaucoup pour ça, parce que c'est intéressant musicalement, à l'inverse d'une bande qui oblige à jouer d'une façon chronométrique, celle-ci permet une véritable interaction entre l'interprète et l'ordinateur. Ce dernier suit vraiment les réactions de l'interprète, mais propose à son tour des variantes, selon ce que l'interprète 
joue; c'est presque de la musique de chambre. Et ça laisse la liberté à l'interprète, selon son humeur, de diriger la pièce dans un sens précis.

R. B. : Il y a donc une part d'improvisation?

B. C. : Oui, à certains endroits de la partition, il y a des moments "plus libres ", mais c'est tout de même très écrit.

R. B. : Vous avez aussi enregistré Pression, d'Helmut Lachenmann, une œuvre où l'interprète doit aussi faire des choix.

B. C. : C'est une partition très graphique. À vrai dire, il ne note pas le résultat à obtenir, mais la manière de faire, le geste. Il y a donc des dessins avec des façons de placer la main, etc.

R. B. : Mais ça, il faut vraiment l'interpréter.

B. C. : Oh oui! C'est l'idée d'une musique concrète instrumentale, donc sans aucun dispositif autre que l'instrument dans toute sa complexité et c'est ça qui est difficile avec cette musique. Au début, on n'entend pas la partition en la lisant, c'est impossible. Il faut travailler la matière pour se rendre compte.

R. B. : Pour l'interprète, c'est un tout autre monde que celui de la partition traditionnelle.

B. C. : Oui et non. En fait, la technique instrumentale n'est forcément pas la même, ce qui se reflète sur la préparation de l'interprète. Mais la réalisation musicale, elle, est identique. On arrive petit à petit à savoir ce que l'on veut entendre, à incarner la musique, à la faire sienne.

R. B. : Mais n'y a-t-il pas, en quelque sorte, une liberté de plus?

B. C. : Je crois qu'il s'agit davantage d'une liberté psychologique pour l'interprète, c'est-à-dire que comme il s'agit d'un type d'écriture qui ne repose pas sur une longue tradition, on a l'impression de pouvoir plus librement apporter sa propre interprétation. Cela dit, pour cette pièce de Lachenmann, qui est particulièrement hors norme en ce qui a trait à la réalisation sonore, on peut comparer les enregistrements qui existent dans le commerce et on entendra la même musique. Effectivement, elle ne sera pas interprétée de la même manière, mais l'on peut constater que c'est quand même très écrit. Cela signifie que c'est surtout de la tradition que l'on se libère. Bien que la pièce date de 1968, elle conserve une certaine fraîcheur à l'interprétation.

R. B. : Ce n'est pas comme une suite de Bach...

B. C. : ... dans laquelle tout le monde sait quelle est la note qui doit venir après.

R. B. : Et puis il y a des interprétations qui sont meilleures que d'autres. Quand on arrive devant ce genre de partition, est-ce qu'on pense à cela?

B. C. : Je trouve qu'on est dans une période intéressante parce qu'il y a eu saturation; tout le monde a eu l'occasion d'exprimer sa manière de faire. Alors 
je crois qu'on revient en terrain neutre où, finalement, tout le monde a bien compris qu'on a le droit d'interpréter la musique comme on la ressent. Il est intéressant de voir que Bach revient dans les programmes de concours alors qu'à un moment, c'était exclu. Il me semble que ça prouve qu'on est un petit peu plus décontracté par rapport à ça. Et il y a aussi beaucoup de jeunes interprètes qui reviennent à l'enregistrement, et sur des instruments modernes, alors qu'il y a dix ans, ça n'aurait pas été toléré.

R. B. : Qu'en est-il justement du choix de l'instrument pour l'interprète?

B. C. : Je pense que l'on choisit son instrument en fonction du répertoire que l'on compte interpréter. C'est difficile de faire de la musique de chambre avec un Stradivarius, parce que l'instrument est tellement présent et a une telle personnalité que les autres instrumentistes peuvent souffrir du décalage. Je ne sais même pas si un quatuor de Stradivarius marcherait bien. Ça deviendrait un quatuor de solistes... L'instrument dont je joue est plutôt un instrument de musique de chambre, justement. Il a un son assez clair, très homogène, par opposition à un son plus capiteux et résonnant. Il est très pratique pour le répertoire avec électronique, plus commode pour la prise de son. Faire la prise de son d'un Stradivarius, c'est tout un problème!

R. B. : Mais vous êtes vous-même davantage un soliste qu'un interprète d'ensemble.

B. C. : Oui. Peut-être par tempérament, mais aussi par la force des choses. Je travaille beaucoup avec l'électronique et, pour le moment, la technologie fait qu'il est beaucoup plus simple d'écrire pour un seul instrument. Mais je n'ai pas le profil du soliste qui enchaîne les concertos de Dvorak, Schumann ou Haydn.

R. B. : Vous êtes plutôt du côté de la création.

B. C. : Oui, et en fait, je dirais plutôt du côté de l'émergence d'un nouveau répertoire.

R. B. : Est-ce une façon d'éviter le face-à-face avec la tradition, de se placer au début plutôt qu'à la suite?

B. C. : Il y a beaucoup d'intérêt pour moi à faire naître une nouvelle partition. C'est un accouchement, quoi. C'est même plus qu'un accouchement; la création de la pièce en concert, c'est la finalité, mais il y a tout le travail en amont. Pas seulement le travail d'apprentissage de l'œuvre, qui est souvent court d'ailleurs parce que les compositeurs sont systématiquement en retard dans l'écriture, à de rares exceptions près. Mais il y a l'aspect social, qui définit bien mieux le rôle de l'interprète dans la création que dans le répertoire. Donner la possibilité à un compositeur d'écrire de la musique, c'est déjà très compliqué, et lorsque l'on a la possibilité de le faire, par une commande, c'est pas mal. Après, c'est le début de la relation avec le compositeur. 
R. B. : Il est vrai que, du point de vue social, ce n'est pas un choix qui facilite la vie...

B. C.: En effet, ce n'est pas le choix le plus simple... Il y a beaucoup moins de possibilités immédiates de concert qu'en jouant des œuvres du répertoire.

R. B. : Et c'est peut-être plus gratifiant pour un interprète de jouer quelque chose que les gens reconnaissent et peuvent comparer.

B. C. : Honnêtement, le concert le plus gratifiant que j'aie eu l'occasion de donner, c'était en 2001, ici à Montréal, à la Chapelle historique du Bon-Pasteur et le retour que j'ai eu de la part du public était tel, que j'ai de la difficulté à l'exprimer; pour moi, dans la création, il y a des moments, par la beauté des œuvres, l'expérience humaine et l'intérêt musicologique, qui m'apportent des plaisirs bien plus importants qu'en jouant du répertoire traditionnel. J'ai choisi de faire de la musique contemporaine en rencontrant la musique de Robert Pascal, par exemple, mais Jonathan Harvey a aussi été très important. Quand j'ai eu l'occasion de les jouer, je ne connaissais pas beaucoup la musique contemporaine, et j'ai peut-être eu de la chance en débutant par des œuvres dont l'esthétique est plus accessible que celle d'un Lachenmann ou d'un Xenakis, par exemple. Ce qui ne veut pas dire, par ailleurs, que je n'ai aucun plaisir à interpréter des œuvres du répertoire, au contraire, j'ai toujours une pratique classique, que j'entretiens quotidiennement en faisant dans mon coin mes suites de Bach et mes sonates de Beethoven. Je pense que pour être performant dans le domaine de la création, de l'art contemporain, il faut avoir une technique classique solide, sinon c'est invivable. Il faut un point d'ancrage. Je ne vais pas pour autant proposer un récital Bach, parce qu'il y a des gens qui le font déjà très bien.

R. B. : Il y aurait peut-être précisément un intérêt particulier à vous voir le faire.

B. C. : Oui, mais c'est trop tôt! J'y viendrai peut-être. Il est vrai que l'on peut se perdre dans la technique contemporaine. Par exemple, lorsque l'on travaille longtemps une œuvre avec des micro-intervalles. Sur les instruments à cordes, les écarts sont dans la main, ils ne sont pas marqués sur l'instrument, donc c'est la main qui structure l'échelle; quand on change ces habitudes, c'est tout un modelage que l'on change et on prend une nouvelle empreinte, alors il faut une petite période d'adaptation pour revenir au répertoire classique. Cela dit, j'ai l'impression depuis que je joue beaucoup de musique contemporaine d'avoir gagné en aisance à l'instrument, parce que je l'ai exploré "géographiquement» dans tous les sens. Et le fait d'aborder des esthétiques parfois difficiles oblige à prendre davantage en charge l'expression musicale, parce qu'on a quand même le souci de faire passer quelque chose. Ce qui fait qu'au moment de revenir au répertoire traditionnel, on est moins «passif», on fait 
1. Concert du 28 novembre 2003. Au programme, des œuvres de Jonathan Harvey (Curve with Plateaux), Dmitry Lybin (La maison au spectre*), Nélio Porto (Dezoito Cantos Indigenas Brasileiros*), Carlo Forlivesi (Più mesto, étude de Montréa/*), Tomi Raïsänen (Time Labyrinth*), Giovanni Mancuso (Farsa Italia $n^{0} 3$, cinque nuovi incidenti diplomatico-politico-musicali*) et Nicolas Gilbert, (Essai sur le désir*), * indiquant les créations. peut-être plus confiance à notre propre sensibilité musicale pour proposer notre interprétation.

R. B. : Vous avez récemment donné un récital à la Chapelle historique du Bon-Pasteur au cours duquel étaient programmées pas moins de six créations que vous aviez travaillées d'assez près avec les compositeurs, prenant même part à la composition'1.

B. C. : Dans ce cas, les compositeurs m'envoyaient des esquisses et je leur faisais des commentaires, mais jamais d'ordre esthétique ou stylistique. J'essaie de reconnaître la qualité du travail au-delà de ces critères, ce qui fait que dans le cas de ce concert, le programme était très éclectique. Si je devais choisir les compositeurs avec qui je travaille en fonction de critères esthétiques ou stylistiques, je jouerais toujours le même genre de pièce. Cependant, il n'est pas exclu que mes commentaires sur la réalisation technique d'une œuvre conditionnent quelque chose dans l'écriture. Mais je ne crois pas influencer suffisamment le compositeur pour m'inscrire à titre de coauteur de l'œuvre. Parfois d'ailleurs les compositeurs voudraient fonctionner de cette façon et c'est moi qui refuse.

R. B. : Quel est votre rapport avec la composition?

B. C. : Vous savez, je pense que certains interprètes sont restés traumatisés par les souvenirs des classes d'écriture, d'analyse, de contrepoint, d'orchestration et de fugue qui mènent à tous ces premiers prix et qui font que l'on se dit que composer, c'est un véritable métier! On conserve un respect face à l'acte de composition. Jusqu'à maintenant, j'ai dû faire une trentaine de créations pour violoncelle seul et, par rapport à mon instrument, je crois qu'il n'est pas exclu que je propose éventuellement quelque chose, mais pour moi. Par exemple, je déplore que tout le répertoire d'étude, du niveau débutant et même jusqu'au niveau intermédiaire, appartienne au $\mathrm{XIX}^{\mathrm{e}}$ siècle. Ça veut dire que l'on apprend à jouer de son instrument selon une esthétique musicale, un genre, une époque, qui conditionnent toute la technique, et je crois que si je devais écrire de la musique, ce serait d'abord pour proposer quelque chose d'autre. Je pense que la création ou, du moins, le répertoire contemporain, a un grand pouvoir pédagogique. On peut y transmettre de nombreuses notions et des réflexes d'apprentissage. Cela dit, écrire une pièce... une œuvre! Je n'en suis pas là. Je préfere pour le moment donner un récital où se côtoient six esthétiques différentes, six langages différents, afin d'offrir un véritable voyage autour de l'instrument. Forcément, pour l'interprète, c'est plus difficile que de se cantonner à une seule vision. On peut penser à un comédien qui doit, dans une pièce, incarner trois personnages différents, par exemple. C'est sans doute plus compliqué que de n'en incarner qu'un seul. Ce qui nous ramène à la question du répertoire traditionnel et contemporain. Je crois qu'il est difficile de tout faire 
très bien, et qu'il n'existe pas vraiment d'interprète qui excelle autant en musique baroque qu'en musique contemporaine. Il s'agit aussi d'affinités, de ce qui nous va plus ou moins, de ce qui nous échappe; souvent c'est le public qui le fait savoir. Prenons deux interprètes ayant des qualités d'interprétation comparables, pourtant une musique donnée ira mieux à l'un qu'à l'autre et ça, ça ne s'explique pas.

R. B. : Ce serait sans doute une question de personnalité encore une fois.

B. C. : Ou de physique! Je pense que pour jouer le Concerto pour violoncelle op. 104 de Dvorak, il faut avoir les épaules que ça exige, on n'en sort pas.

R. B. : Parlez-moi de votre résidence actuelle à Montréal.

B. C. : J'ai obtenu une bourse du ministère des Affaires étrangères de la France en partenariat avec le Conseil des arts et des lettres du Québec et l'Office franco-québécois pour la jeunesse. Ils se sont mis à trois pour gérer ce programme d'échange de résidences qui permet d'accueillir ici des artistes français pour une durée de six mois, afin de réaliser un projet artistique. Le mien consistait à offrir ma disponibilité d'interprète à des compositeurs, parce que ces résidences sont généralement réservées aux créateurs : plasticiens, architectes, vidéastes, compositeurs, etc., alors que moi, je ne suis qu'interprète. Comme j'aime collaborer de près avec les compositeurs, j'ai donc offert de travailler à de nouvelles pièces, de les jouer en concert et de les enregistrer. Quand ça a été accepté, évidemment, je l'ai fait savoir et j'ai envoyé des invitations un peu partout, dans les universités et conservatoires, studios de musique contemporaine, etc., environ 1000 courriels en tout et j'ai reçu 500 réponses! dans le lot, 70 compositeurs ont envoyé des propositions concrètes de collaboration et, après étude, j'en ai gardé 14 . Six œuvres pour violoncelle acoustique ont été créées à la Chapelle historique le 28 novembre 2003. Les huit autres seront créées à Lyon à la biennale Musiques en scène 2004; ce sont des œuvres incorporant l'électroacoustique pour lesquelles je serai épaulé par le GRAME.

R. B. : Permettez-moi de revenir sur une phrase que vous avez dite en parlant du programme de résidence; vous avez dit « je suis seulement interprète ", par opposition à ceux qui sont « créateurs »...

в. С. : Oui, c'est vrai que je fais tellement de création... Je suis certainement plus à l'aise dans l'invention pure, et ça m'amènera peut-être un jour à la composition, que dans la restitution exacte d'une tradition orale. J'aime inventer des projets et de nouvelles manières de travailler et c'est pourquoi je me suis dirigé, à une époque où ce n'était pas «naturel », vers l'électronique.

R. B. : Il y a un grand courant de musiques improvisées, et il arrive de plus en plus souvent que l'on puisse voir un instrumentiste qui donne un récital d'improvisation en solo. Cela pourrait-il vous attirer? 
B. C. : Oui, c'est la même question que pour la composition... Jonathan Harvey disait : "J'imagine ma musique aussi fraîche qu'une improvisation, mais sans qu'elle contienne pour autant aucune note déplacée.» J'adore cette phrase, parce que je crois beaucoup à l'écriture et je crois beaucoup à la spontanéité de l'improvisation, mais je m'en méfie. J'ai assisté assez souvent à des concerts d'impro, mais peut-être n'était-ce pas les bons... Peut-être trop souvent des concerts de musiciens classiques s'essayant à l'improvisation... Il y a des improvisations qui sonnent comme de mauvaises compositions... Et de mauvaises compositions qui sonnent comme des improvisations!

Je pense que lorsque l'on joue avec de l'électronique, la définition du mot «interprète » a les contours un petit peu plus flous, parce qu'à partir du moment où l'on est dans le domaine de l'interactivité, il y a improvisation, forcément. On n'est pas loin de la composition parfois, parce qu'il faut prendre en charge la responsabilité musicale, bien plus que dans le cas de l'exécution d'un texte. Maintenant, l'improvisation pure, ce n'est pas mon domaine. Je serais partant pour des tentatives, mais je n'ai pas trouvé pour le moment, au-delà de ce que demandent certaines partitions qui comprennent des situations d'improvisation, de déclencheur qui puisse m'attirer assez vers ce type de musique. Je n'y serais pas à l'aise. Je suis de plus en plus préoccupé par la question de la forme. À force d'avoir croisé toutes ces créations, je me rends compte que je deviens de plus en plus soucieux du rendu des œuvres et que pour bien y parvenir, il faut vraiment avoir une idée claire de la forme de l'œuvre. Je me rends compte également que plus le compositeur et l'interprète travaillent ensemble et plus le résultat est intéressant. Systématiquement, lorsque l'on travaille ensemble, la partition évolue beaucoup, alors que si on ne se retrouve que pour le concert, la partition n'a pas eu le temps d'évoluer et, une fois qu'elle est créée, il est trop tard, elle est née. Le travail avec le compositeur permet à l'interprète de se sentir mieux avec l'œuvre, d'y être à l'aise et d'acquérir une confiance qui est extrêmement communicative, une authenticité de fait. Je tiens quand même à signaler que la première interprétation d'une œuvre est rarement la meilleure. Lorsque l'on reprend une œuvre, même sans l'avoir retravaillé, elle a mûri.

R. B. : C'est dommage pour bien des pièces qui ne sont entendues qu'une fois...

B. C. : Voilà. C'est pourquoi il est important de travailler avec le compositeur, pour ne pas rater la création. Parce qu'après, on aura du mal à revenir dedans. Ce n'est pas un acte facile... 